\title{
Variance Function Regressions for Studying Inequality
}

\section{Citation}

Western, Bruce and Deirdre Bloome. 2009. Variance function regressions for studying inequality. Working paper, Department of Sociology, Harvard University.

\section{Permanent link}

http://nrs.harvard.edu/urn-3:HUL.InstRepos:2645469

\section{Terms of Use}

This article was downloaded from Harvard University's DASH repository, and is made available under the terms and conditions applicable to Open Access Policy Articles, as set forth at http:// nrs.harvard.edu/urn-3:HUL.InstRepos:dash.current.terms-of-use\#OAP

\section{Share Your Story}

The Harvard community has made this article openly available.

Please share how this access benefits you. Submit a story.

\section{Accessibility}




\title{
Variance Function Regressions for Studying Inequality
}

\author{
Bruce Western $^{1} \quad$ Deirdre Bloome \\ Harvard University
}

January 2009

\footnotetext{
${ }^{1}$ Department of Sociology, 33 Kirkland Street, Cambridge MA 02138. E-mail: western@wjh.harvard.edu. This research was supported by a grant from the Russell Sage Foundation.
} 


\begin{abstract}
Regression-based studies of inequality model only between-group differences, yet often these differences are far exceeded by residual inequality. Residual inequality is usually attributed to measurement error or the influence of unobserved characteristics. We present a regression that includes covariates for both the mean and variance of a dependent variable. In this model, the residual variance is treated as a target for analysis. In analyses of inequality, the residual variance might be interpreted as measuring risk or insecurity. Variance function regressions are illustrated in an analysis of panel data on earnings among released prisoners in the $\mathrm{Na}$ tional Longitudinal Survey of Youth. We extend the model to a decomposition analysis, relating the change in inequality to compositional changes in the population and changes in coefficients for the mean and variance. The decomposition is applied to the trend in US earnings inequality among male workers, 1970 to 2005.
\end{abstract}


In studying inequality, we can distinguish differences between groups from differences within groups. Sociological theory usually motivates hypotheses about between-group inequality. For these hypotheses, interest focuses on differences in group averages. For example, theories of labor market discrimination predict whites earn more than blacks, and men earn more than women. Human capital theory explains why college graduates average higher earnings than high school dropouts. Such theories are often tested with a regression where differences in groups means are quantified by regression coefficients.

Although theory usually focuses on between-group differences, withingroup variance also contributes to inequality. Within-group inequality can be measured by the residual variance of a regression. Typically the residual is viewed as unexplained, and its variation is not treated as substantively interesting. Although it is often overlooked, residual heterogeneity may vary in substantively important ways. Some groups may be more insecure than others, or vary more in unobserved characteristics. The structure of within-group inequality may be especially important for sociological analysis where the residual variance often greatly exceeds the the between-group variance.

We present a statistical model of inequality that captures the effects of covariates on within-group and between-group inequality. Called a variance-function regression, the model features separate equations for the mean and variance of the dependent variable. Regression coefficients for the mean and variance can be estimated with specialized calculations, though we show that they are well-approximated in large samples with standard software.

Though variance function regression have a long history in economet- 
rics and statistics (Park 1966; Harvey 1976; Cook and Weisberg 1983), we use them to make three contributions to the sociological analysis of inequality. First, from a substantive viewpoint, a statistical model for the residual variance challenges sociological theory to explain not just average differences between groups, but also differences in the heterogeneity of groups. Large coefficients for the residual variance indicate large differences in within-group inequality. Below we motivate interest in these differences in within-group inequality with theories of economic insecurity.

Second, a few studies have analyzed variation in the residual variance, but only as a function of macro predictors (like metro areas or occupations), and only using ad hoc methods for estimation. We follow the statistical literature by writing a likelihood that includes regression coefficients for the conditional mean and the variance. This approach allows macro and micro predictors for the residual variation and enables the joint estimation of regression coefficients with smaller mean squared error than ad hoc approaches. We apply the model in an analysis of panel data to test the hypothesis that men released from prison experience greater earnings insecurity (greater variance) in addition to the well-documented decline in average earnings.

Finally, we apply the model to a standard decomposition of the change in variance. This extension of the decomposition analysis offers a simple way of studying the effects of individual independent variables on changes in inequality. In our approach, changes in inequality may result from: (1) changes in the distribution of an independent variable, (2) changes in means across levels of an independent variable, or (3) changes in variances across levels of an independent variable. We also describe a Bayesian 
approach to estimation that yields inferences for nonstandard quantities from the variance decomposition, whose sampling uncertainty is usually ignored. These methods are illustrated in an analysis of the trend in US earnings inequality using data from the March Current Population Survey.

\section{Between-Group and Within-Group Inequality in Sociology}

In a very general sense, sociologists are pervasively interested in betweengroup inequality. Most claims about variability in a population describe average differences between groups. Of course, not all studies of betweengroup difference are framed as analyses of inequality. But where inequality is the focus, it is generally conceived in between-group terms.

The emphasis on between-group inequality seems clearest in theories emphasizing categorical inequalities-inequalities between categorically defined groups (Tilly 1998; Massey 2007). In these accounts, out-groups receive less because in-groups monopolize resources and restrict access to opportunities. Average differences in incomes, well-being, and mobility emerge as a result. The labor market theory of discrimination exemplifies an account of categorical difference whose main empirical implications are for between-group inequality. Research on racial and gender discrimination thus estimates black-white differences, or female-male differences in earnings, typically controlling for a large number of confounding factors (Cancio, Evans, and Maume 1996; Budig and England 2001).

Regression provides a convenient framework for this analysis, where the regression coefficients describe differences in group means. Of course, regression also describes between-group differences with continuous predictors. In this case, groups are defined across the fine gradations of the continuous variable. 
Although between-group differences dominate sociological thinking about inequality, the regression model also includes a term for withingroup differences. Write the regression for observation $i$,

$$
y_{i}=b_{0}+b_{1} x_{i}+e_{i},
$$

with expected value $\hat{y}_{i}=b_{0}+b_{1} x_{i}$. With errors, $e_{i}$, uncorrelated with the predictors, $x_{i}$, inequality in $y_{i}$, measured by the variance, can be expressed as the sum of the variance between groups and the variance within groups,

$$
\begin{aligned}
& V\left(y_{i}\right)=\begin{array}{c}
V\left(\hat{y}_{i}\right) \\
\uparrow
\end{array} \quad+\quad \begin{array}{c}
\uparrow\left(e_{i}\right) . \\
\uparrow
\end{array} \\
& \text { Between Within } \\
& \text { Group Group }
\end{aligned}
$$

In a least squares regression, the empirical residuals are uncorrelated with $x_{i}$ by construction, so the variance of $y_{i}$ mechanically equals the sum of the residual variance and the variance of predicted values for the $y_{i}$. The residual variance, $V\left(e_{i}\right)$, may reflect measurement error rather than an underlying social process. Often, however, residuals are viewed as capturing real but substantively uninteresting variation. For example, Blau and Duncan $(1967,174)$ remark that residuals reflect a (thankfully) unpredictable social world, but the magnitude of residuals is unimportant for understanding inequalities in educational attainment or occupational status. "The relevant question about the residual," they write, "is not really its size at all, but whether the unobserved factors it stands for are properly represented as being uncorrelated with measured antecedent variables" (Blau and Duncan 1967,175). From this perspective, residuals are not intrinsically interesting, but may be helpful for discovering omitted variables. 
In contrast to Blau and Duncan (1967), residual variability may be a substantively important difference between groups. For example, among children at age 10, boys are over-represented in the top tail of the distribution of measured intelligence, and average slightly higher scores than girls on intelligence tests. However, the over-representation of boys among highly intelligent children is due significantly to the greater dispersion of boy's scores (Arden and Plomin 2006). Here, the salient difference between boys and girls is not just the location of their test score distributions, but the spread of those distributions too. Comparing distributions across groups helps enrich the account of group differences beyond stylized facts about the difference of means.

In research on inequality, the substantive significance of the residual was considered in Jencks's discussion of the income distribution (Jencks et al. 1972). For Jencks, the large residual variance in regressions on incomes results from workers' unmeasured skills and luck. An appealing personality and athletic talent are offered as examples of unmeasured skills. Luck might include "chance acquaintances who steer you to one line of work rather than another, the range of jobs that happen to be available in a particular community when you are job hunting, ... and a hundred other unpredictable accidents" (Jencks et al. 1972, 227). The influence of luck on income inequality might be reduced through insurance, Jencks argues, suggesting that luck might also be described as income insecurity.

A similar interpretation of the residual variance is provided in recent research on US income inequality. The growth of US inequality in the 1980s and 1990s was marked by a steady increase in the residual variance in regressions of earnings on experience and schooling. Labor economists argued that growth in within-group inequality reflected rising returns to 
unobserved skills and compositional changes which multiplied the numbers of high-skill workers with highly variable incomes (Katz and Murphy 1992; Lemieux 2006). Others, sociologists and economists, countered that increasing within-group inequality resulted from workers' increasing exposure to competitive forces in the labor market (DiNardo, Fortin, and Lemieux 1996; Massey 2007). Institutions like the minimum wage, labor unions, and the career ladders of large firms made income more secure and sheltered wages from market forces. As these institutional protections eroded through the 1970s and 1980s, within-group inequality in earnings increased. McCall (2000) thus refers to the "deinstitutionalization" of the American labor market, and Sørensen (2000) points to the elimination of labor market rents as a source of increasing income insecurity. Consistent perhaps with rising returns to unobserved skills and rising economic insecurity, increased within-group inequality has also been found to be a driver of inequality in China during the period of rapid market transition from the late 1980s to the mid-1990s (Hauser and Xie 2005). Theories of unobserved skill and labor market deinstitutionalization depart from accounts of between-group inequality by claiming that the residual variance is larger for some groups than others.

Sociological research on within-group inequality has taken residual standard deviations and other measures of within-group inequality as dependent variables for regression. McCall's (2000) study of labor market institutionalization took a two-stage approach, first regressing log incomes on demographic covariates. The residuals from this first-stage regression were used to form residual standard deviations for metro areas which were then regressed on metro-level measures of employment and industry-structure. Sørensen and Sorenson (2007) also took a two-stage 
approach in their analysis of Danish data. Obtaining residuals from a regression on log wages, they calculated log residual standard deviations for local areas which were regressed on measures of the competitiveness of local product markets. In contrast to the small-area analysis, Kim and Sakamoto (2008) regress Gini indexes of occupational wage inequality on occupation-level predictors. In all these analyses, within-group inequality is viewed as the product of macro-level predictors. Thus variables measured at the level of occupational groups or metro areas, for example, have been written as predictors of within-group inequality. Estimation proceeds in two stages where residuals are calculated from a first stage regression, and residual dispersion is regressed on macro predictors in the second stage.

We next introduce a model that jointly estimates the effects of predictors on between-group and within-group inequality. Jointly fitting withingroup and between-group effects takes us beyond macro-level studies of within-group inequality in two ways. First, our model allows for the effects of micro-level and macro-level variables on within-group inequality. Second, by jointly estimating between-group and within-group coefficients, inferences about one set of coefficients also incorporate uncertainty about the other.

Formalizing ANd Estimating the Model

For observation $i(i=1, \ldots, n)$ on a dependent variable, $y_{i}$, the variance function regression writes the mean, $\hat{y}_{i}$, and the variance, $\sigma_{i}^{2}$, both as a 
function of covariates,

$$
\begin{aligned}
\hat{y}_{i} & =\boldsymbol{x}_{i}^{\prime} \beta \\
\log \sigma_{i}^{2} & =\boldsymbol{z}_{i}^{\prime} \boldsymbol{\lambda},
\end{aligned}
$$

where $x_{i}$ is a $K \times 1$ vector of covariates for the mean, and $z_{i}$ is a $J \times 1$ vector of covariates (possibly equal to $x_{i}$ ) for the variance. ${ }^{1}$ In this this model, a coefficient $\beta_{k}$ has the usual interpretation, describing the average difference in $y$ associated with a one unit change on an independent variable, $x_{k}$. Early proposals viewed the variance coefficients, $\lambda$, as a diagnostic for heteroscedasticity (Cook and Weisberg 1983). In studying inequality, the $\lambda$ coefficients are substantively interesting, describing the association of covariates with within-group inequality. A variance coefficient $\lambda_{j}$ is interpreted as the difference in the log variance associated with a unit change in $z_{j}$. We are familiar with a single observation, $y_{i}$ having a conditional mean given observations on independent variables, $\boldsymbol{x}_{i}$, but the idea of a conditional variance for a single observation may be less intuitive. In this case, the model describes not where $y_{i}$ will fall on average, but how far $y_{i}$ will fall from this average value, given $z_{i}$. From a substantive viewpoint, the model formalizes the idea that values of $x_{i}$ and $z_{i}$ are associated not just with high or low values of $y_{i}$ but are also associated with the variability or unpredictability of $y_{i}$.

The variance function model clearly relaxes some of the assumptions of the usual linear regression. Unlike the constant variance linear regression, the variance function model is heteroscedastic, allowing the residual variance to depend on covariates. Though the variance function regression is

\footnotetext{
${ }^{1}$ It is often useful to transform the dependent variable to the log scale yielding a scale invariant measure of inequality, the variance of $\log y_{i}$. We discuss this in greater detail below.
} 
relatively general, the model assumes that the mean and variance are linear functions of covariates. The mean and variance of $y_{i}$ are also assumed to be independent, conditional on $\boldsymbol{x}_{i}$ and $z_{i}$. The $y_{i}$ are also assumed to be independent. Each of these assumptions could be relaxed by allowing for a more general functional form for the regression relationships, or by specifying a more complex structure for the covariance matrix of $y_{i}$. The linearity of the mean and variance functions could be relaxed by adding nonlinear terms to the regression or by writing the mean and variance as nonlinear in the parameters. The independence assumption for $y_{i}$ could be relaxed by allowing cross-correlation terms in the covariance matrix or by adding random effects in the mean regression. Correlations between the mean and variance could be allowed by writing the variance as a function of $\hat{y}_{i}$.

Variance function regressions have a relatively long history in statistics and econometrics and were originally motivated by parametric tests for heteroscedasticity (Anscombe 1961; Park 1966; Cook and Weisberg 1983). Joint maximum likelihood estimation of the mean and variance coefficients was developed in subsequent studies (Harvey 1976; Aitkin 1987; Verbyla 1993). Though we know of no research with these models in sociology, there are recent applications in the sciences and social sciences which study the effects of covariates on the variance. Agricultural studies have recently examined variability in the survival rates of fish populations, and modelled the variance of crop yields (Minto, Myers, and Blanchard 2008; Edwards and Jannink 2006). In the social sciences, economists have studied predictors of retail prices and political scientists have analyzed the variance of vote choice in referenda (Lewis 2008; Selb 2008). In all these studies, the structure heteroscedasticity was of key scientific 
interest.

\section{Estimation}

Several methods have been proposed to estimate the variance function regression. First, a simple two-stage approach uses standard software to fit a linear regression, then a generalized linear model to the transformed residuals (Nelder and Lee 1991). For this method:

1. Estimate $\beta$ with a linear regression of $y_{i}$ on $x_{i}$. Save the residuals, $\hat{e}_{i}=y_{i}-\boldsymbol{x}_{i}^{\prime} \hat{\boldsymbol{\beta}}$, where $\hat{\boldsymbol{\beta}}$ is the least squares estimate.

2. Estimate $\lambda$ with a gamma regression of the squared residuals, $\hat{e}_{i}^{2}$, on $z_{i}$, using a log link function.

The gamma regression is a type of generalized linear model for positive right-skewed dependent variables. The regression can be fit with standard software such as the glm command in Stata or GENMOD in SAS. The point estimates with this method are consistent, but the standard errors are incorrect. In particular, the standard errors for the estimates of $\lambda$ take no account of the uncertainty in $\beta$, and estimates of $\beta$ are inefficient because they ignore heteroscedasticity in $y_{i}$.

Second, maximum likelihood estimates are obtained by iterating the two stage method (Aitkin 1987). In addition to the assumptions above, if we assumed that $y_{i}$ is conditionally and independently normal with mean $\hat{y}_{i}$ and variance $\sigma_{i}^{2}$, the contribution of observation $i$ to the log likelihood is

$$
\begin{aligned}
L\left(\beta, \lambda ; y_{i}\right) & =-\frac{1}{2}\left[\log \left(\sigma_{i}^{2}\right)+\left(y_{i}-\hat{y}_{i}\right)^{2} / \sigma_{i}^{2}\right] \\
& =-\frac{1}{2}\left[\boldsymbol{z}_{i}^{\prime} \boldsymbol{\lambda}+d_{i} \exp \left(-\boldsymbol{z}_{i}^{\prime} \boldsymbol{\lambda}\right)\right]
\end{aligned}
$$


where $d_{i}$ is the squared residual, $\left(y_{i}-\hat{y}_{i}\right)^{2}$. To obtain the maximum likelihood estimates:

1. Fit a linear regression of $y_{i}$ on $\boldsymbol{x}_{i}$, yielding the estimated coefficients, $\hat{\boldsymbol{\beta}}$, and residuals, $\hat{e}_{i}=y_{i}-\boldsymbol{x}_{i}^{\prime} \hat{\beta}$.

2. Fit a gamma regression with a $\log \operatorname{link}$ of $\hat{e}_{i}^{2}$ on $z_{i}$, yielding current estimates $\hat{\lambda}$. Save the fitted values, $\hat{\sigma}_{i}^{2}=\exp \left(z_{i}^{\prime} \hat{\lambda}\right)$.

3. Fit a weighted linear regression of $y_{i}$ on $\boldsymbol{x}_{i}$, with weights, $1 / \hat{\sigma}_{i}^{2}$. Update the residuals, $\hat{e}_{i}$, and evaluate the log likelihood.

4. Iterate steps 2 and 3 to convergence, updating $\hat{\beta}$ and $\hat{e}_{i}$ from the linear regression, and $\hat{\lambda}$ and $\hat{\sigma}_{i}^{2}$ from the gamma regression.

Like many generalized linear models, the gamma regression is commonly fit by iteratively weighted least squares. If coefficients from the previous iteration are used as start values, computation can be speeded by fitting just one step of the gamma regression (Smyth, Huele, and Verbyla 2001, 164). Like the two-stage estimator, ML estimation can be performed with standard software for generalized linear models. (A Stata macro is given in Appendix 1.)

The maximum likelihood estimator may perform poorly in small samples because variance estimation does not adjust for degrees of freedom and a biased score vector is used for estimation. A restricted maximum likelihood (REML) estimator based on the marginal likelihood for $\lambda$ produces estimates that are less biased in small samples (Smyth, Huele, and Verbyla 2001). Unlike the two-stage and ML estimation, REML estimation requires specialized calculations. Smyth (2002) describes an efficient REML algorithm which has been implemented in $\mathbf{R}$. 
The variance-function regression can also be placed in a Bayesian framework. Bayesian analysis offers two advantages. First in small samples, the $\lambda$ coefficients in the variance equation may be skewed and inference based on the normal distribution will be inaccurate. Nonnormality in the posterior distribution will be revealed by simulation from the Bayesian posterior distribution. Second, some analyses, like the variance decomposition below, will focus not on the model coefficients themselves, but on nonlinear functions of the coefficients. Output from the Bayesian posterior simulation can be used to construct inferences for these functions of model parameters.

The Bayesian model combines the normal likelihood for $y_{i}$ with a prior distribution for the coefficients, $\beta$, and a hierarchical prior for the variance coefficients, $\lambda$. For a dependent variable, $y_{i}$, with predictors $\boldsymbol{x}_{i}$ for the mean and $z_{i}$ for the variance, the Bayesian model can be written:

$$
\begin{aligned}
& y_{i} \sim N\left(\hat{y}_{i}, \sigma_{i}^{2}\right), \text { where } \\
& \hat{y}_{i}=\boldsymbol{x}_{i}^{\prime} \beta \text { and } \log \sigma_{i}^{2}=z_{i} \lambda
\end{aligned}
$$

with prior distributions,

$$
\begin{aligned}
\beta & \sim N(\boldsymbol{b}, \boldsymbol{V}) \\
\lambda & \sim N(\boldsymbol{g}, \boldsymbol{U}) \\
U_{j j} & \sim \operatorname{Gamma}^{-1}\left(u_{0}, u_{1}\right)
\end{aligned}
$$

A noninformative prior sets the prior mean vectors, $\boldsymbol{b}$ and $\boldsymbol{g}$, all to zero. The $K \times K$ prior covariance matrix, $V$, is diagonal with large prior variances, say $10^{6}$. To help ensure the sample data dominates estimation of the variance coefficients, $\lambda$ is given a hierarchical prior. The $J \times J$ covariance matrix, $\boldsymbol{U}$, is diagonal and the prior variances follow an inverse Gamma 
distribution with hyperparameters, $u_{0}=.001$ and $u_{1}=.001$. (We also experimented with a nonhierarchical prior on $\lambda$ though this approach performed poorly in small samples.) The Bayesian model can be estimated with MCMC software like BUGS. (BUGS code is given in Appendix 2.)

\section{Comparing Estimation Methods}

The four estimation methods-two-step, ML, REML and Bayes-vary in ease of application. The two-step and ML methods can be fit with standard software, while REML and Bayesian estimation require specialized calculations. Do the four methods perform comparably?

We performed a Monte Carlo experiment to compare two-stage, ML, and REML, and Bayesian estimators. This experiment was based on one covariate, $\boldsymbol{x}_{q}$, a vector consisting of $q$ replicates of $\boldsymbol{x}^{\prime}=[1,2, \ldots, 10]$. The dependent variable, $y_{i}$ was generated from,

$$
y_{i} \sim N\left(\hat{y}_{i}, \sigma_{i}^{2}\right),
$$

where $\hat{y}_{i}=.1+.1 \times x_{q i}$, and $\sigma_{i}^{2}=\exp \left(.3+.3 \times x_{q i}\right)$. We generated $y_{i}$ for $q=5$ and 50, corresponding to sample sizes $n=50$ and 500. The four estimators were applied to each data set of $x_{q i}$ and $y_{i}$. Estimates were obtained for 2000 replications at each sample size.

The experimental results are reported in Table 1. With the small sample, $n=50$, biases for all estimators are generally modest. However, for the intercept of the variance function, $\lambda_{0}$, bias of the MLE is larger than for the other estimators by a factor of 2 to 5 . Though we might expect the prior distribution to influence estimates in small samples, bias in the Bayesian analysis is similar to that for REML. The advantages of likelihood-based approaches (including Bayes) can be seen by comparing the sampling vari- 
Table 1. Results from a Monte Carlo experiment for two-stage, ML, REML, and Bayesian estimators of a variance-function regression.

\begin{tabular}{|c|c|c|c|c|}
\hline & $\beta_{0}$ & $\beta_{1}$ & $\lambda_{0}$ & $\lambda_{1}$ \\
\hline \multicolumn{5}{|c|}{ Bias of point estimates, $n=50$} \\
\hline Two-stage & -.024 & .004 & -.017 & -.009 \\
\hline ML & -.009 & .001 & -.100 & .003 \\
\hline REML & -.007 & .000 & -.045 & .000 \\
\hline Bayes & -.011 & -.001 & -.042 & .006 \\
\hline \multicolumn{5}{|c|}{ Sampling variance of point estimates, $n=50$} \\
\hline Two-stage & .538 & .030 & .202 & .005 \\
\hline ML & .312 & .018 & .212 & .005 \\
\hline REML & .312 & .018 & .213 & .005 \\
\hline Bayes & .312 & .018 & .213 & .005 \\
\hline \multicolumn{5}{|c|}{ Coverage rate of $95 \%$ interval, $n=50$} \\
\hline Two-stage & .986 & .914 & .918 & .926 \\
\hline ML & .945 & .939 & .906 & .917 \\
\hline REML & .939 & .940 & .945 & .945 \\
\hline Bayes & .956 & .960 & .962 & .969 \\
\hline \multicolumn{5}{|c|}{ Bias of point estimates, $n=500$} \\
\hline Two-stage & .002 & -.001 & -.002 & -.001 \\
\hline ML & .001 & -.001 & -.011 & .000 \\
\hline REML & .001 & -.001 & -.004 & .001 \\
\hline Bayes & -.001 & -.001 & -.001 & .000 \\
\hline \multicolumn{5}{|c|}{ Sampling variance of point estimates, $n=500$} \\
\hline Two-stage & .053 & .003 & .020 & .001 \\
\hline ML & .029 & .002 & .020 & .001 \\
\hline REML & .029 & .002 & .020 & .001 \\
\hline Bayes & .029 & .002 & .020 & .001 \\
\hline \multicolumn{5}{|c|}{ Coverage rate of $95 \%$ interval, $n=500$} \\
\hline Two-stage & .989 & .920 & .938 & .944 \\
\hline ML & .950 & .944 & .938 & .942 \\
\hline REML & .950 & .944 & .943 & .943 \\
\hline Bayes & .950 & .945 & .958 & .950 \\
\hline
\end{tabular}

Note: For each sample size, $n=50$ and $n=500,2000$ Monte Carlo samples were drawn. BUGS code for the Bayesian estimation is reported in the Appendix. 
ance of point estimates. The sampling variance of $\beta$ with the two-stage estimator is nearly twice as large as the other methods, unsurprising given the inefficiency of OLS in the presence of heteroscedasticity. The performance of inferential statistics is measured by how frequently nominal confidence intervals cover the known regression coefficients. Nominal confidence intervals for the two-stage and ML estimator are often too optimistic in small samples, overstating coverage rates. REML and Bayes yield uniformly more accurate frequentist inference in small samples. REML standard errors are slightly optimistic, and Bayesian standard errors are slightly pessimistic, with nominal intervals being long, given their coverage rates.

The performance of all the estimators improves as sample size gets large. With $n=500$, there is very little bias in the point estimates of either $\beta$ or $\lambda$. As sample sizes increase by a factor of 10 , sampling variances decrease in similar proportion. The two-stage estimates of $\beta$ (OLS estimates) remain relatively inefficient compared to the other methods that account for heteroscedasticity. The sampling variance of all estimators are similar for the variance coefficients, $\lambda$. Standard errors and confidence intervals also tend to be more accurate with large-sample sizes. Coverage rates for the two-stage and ML estimators are slightly optimistic on average. By contrast, nominal coverage rates for REML and Bayesian intervals are almost exactly equal to their true rates.

The Monte Carlo experiments show that Bayesian and REML estimators, at these parameter values, perform better in small samples than ML and two-stage methods. With $n=50$, the two-stage estimator provides poor estimates of the mean coefficients, $\beta$, and maximum likelihood poorly estimates the variances coefficients, $\lambda$. The performance of all estima- 
tors improves as sample size gets large, for $n=500$. The two-stage estimator is clearly the most inefficient. It can be improved with an additional weighted least squares step to estimate $\beta$ with weights $1 / \hat{\sigma}_{i}^{2}$, estimated from the gamma regression on the log of the squared OLS residuals. Bayes and REML perform consistently better than the other two methods. Though the computational cost of Bayesian estimation is far higher than all the other methods, outputs from the Bayesian posterior simulation allows inference for a variety of quantities derived from the parameter estimates. These inferences are illustrated in the decomposition below.

\section{Application I: InCARCERATION ANd EARNings Insecurity}

In the context of increasing incarceration rates in the United States, researchers have recently examined the effects of imprisonment on the earnings and employment of ex-offenders (Kling 2006; Western 2002; Pager 2003). Western (2006) examined the effects of incarceration on annual earnings, using panel data from the 1979 cohort of the NLSY (NLSY79). Previous research has generally studied whether earnings decline, on average, after an offender is released from prison. Because the formerlyincarcerated mostly find work in the secondary sector of the labor market in which job tenure is relatively short, incarceration likely affects not just the average level of earnings, but also the variability of earnings.

We study this hypothesis with a variance function regression that models the mean and variance of log earnings for men who go to prison. We analyze data on annual earnings from the NLSY79 for male respondents who are interviewed in prison at some time from 1983 to 2000. Descriptive statistics show that 517 male respondents were interviewed at least once in prison after 1983 (Table 1). Log annual earnings is slightly lower 
Table 1. Descriptive statistics for an analysis of the effects of incarceration on annual earnings among young men interviewed in prison, NLSY79, 1983-2000.

\begin{tabular}{lcc}
\hline \hline & $\begin{array}{c}\text { Before } \\
\text { Incarceration }\end{array}$ & $\begin{array}{c}\text { After } \\
\text { Incarceration }\end{array}$ \\
\hline Log annual earnings & 9.10 & 9.05 \\
Variance of log earnings & 1.30 & 1.74 \\
Work experience (weeks per year) & 30.76 & 25.06 \\
Years of schooling & 10.78 & 10.92 \\
\hline Respondent-years & 1718 & 1970 \\
Number of respondents & \multicolumn{2}{c}{517} \\
\hline
\end{tabular}

on average after respondents are released from prison. The variance of earnings is also larger after incarceration. Key covariates of incarceration include work experience and years of schooling. Work experience is measured as the cumulative mean of average weeks worked in a year. Work experience drops significantly among ex-prisoners. Years of schooling is slightly higher for ex-prisoners reflecting additional education obtained after release from prison. The descriptive statistics also indicate that each NLSY respondent contributes an average of 7 interviews to the sample.

In this analysis we fit fixed effects to the model for the mean to account for unobserved heterogeneity across respondents. Fixed effects are fit by subtracting the respondent-level means from the dependent and independent variables. We also estimate the residual variance as a function of the mean-deviated independent variables. Parameterized this way, the intercept term from the variance function regression approximates the average log residual variance. The variance function coefficients will vary depending on whether the mean-deviated or raw predictors are used.

The effects of incarceration on earnings are captured by two predictors. The effect of interest-the effect of incarceration on the earnings of those released from prison-is estimated with a dummy variable that 
Table 2. Variance function regression results for a fixed effects analysis of log earnings among incarcerated men, NLSY79, 1983-2000. (Standard errors in parentheses.)

\begin{tabular}{lcccc}
\hline \hline & \multicolumn{2}{c}{ REML } & \multicolumn{2}{c}{ Bayes } \\
\cline { 2 - 5 } & $\beta$ & $\lambda$ & $\beta$ & $\lambda$ \\
\hline Intercept & .086 & -.147 & .085 & -.149 \\
& $(.018)$ & $(.027)$ & $(.018)$ & $(.025)$ \\
Previously Incarcerated & -.326 & .464 & -.329 & .435 \\
& $(.056)$ & $(.086)$ & $(.056)$ & $(.078)$ \\
Currently Incarcerated & -.460 & .196 & -.462 & .178 \\
& $(.050)$ & $(.076)$ & $(.051)$ & $(.071)$ \\
Years of Schooling & .041 & -.119 & .038 & -.107 \\
& $(.032)$ & $(.050)$ & $(.032)$ & $(.042)$ \\
Work Experience & .010 & -.017 & .010 & -.017 \\
& $(.003)$ & $(.004)$ & $(.003)$ & $(.004)$ \\
\hline
\end{tabular}

Note: Model for the mean and variance of log annual earnings also included the effects of age, local area unemployment, enrollment status, region, urban residence, drug use, union membership, public sector employment and 6 industry categories. $N=3,688$, from 517 respondents.

scores zero in all years up to release from prison, and one thereafter. Because self-reported earnings tend to be very low in the years a respondent is incarcerated, we also introduce a dummy variable indicating current incarceration status.

Like the Monte Carlo results, REML and Bayesian estimates of the regression results are very similar in the NLSY (Table 2). Our interest focuses on the mean and variance of log earnings for men who have been incarcerated. The REML estimate indicates incarceration reduces average annual earnings by about 30 percent $\left(1-e^{-.326}=.278\right)$. The Bayesian estimate of this effect and its standard error are almost identical. The variance function coefficients show that the residual variance in log earnings is higher after incarceration than before. With the REML estimate, the 
residual variance of earnings rises by about 60 percent $\left(e^{0.464}=1.590\right)$. The Bayesian point estimate is somewhat smaller, but tells a similar substantive story, that men who have been incarcerated experience greater variability in earnings.

Against the effects of incarceration, schooling and work experience, which are associated with higher average earnings, are also associated with less earnings variability for this sample of predominantly low-skill, crime-involved, men. Point estimates suggest that each year of schooling is associated with a 10 percent reduction in the residual variance of earnings inequality. Each week of work experience is associated with a 1.7 percent reduction in the variability of earnings.

In sum, in this sample of incarcerated NLSY respondents, more skilled respondents tend to have higher than average earnings and less earnings variability. The very low-skilled, including the formerly-incarcerated, have lower than average earnings and greater variability in earnings. These results indicate greater earnings insecurity among the less-skilled and less-experienced.

\section{DeCOMPOSING TRENDS IN INEQUALITY}

While the parameters of the variance function regression may be substantively interesting, they can also be used to study trends in inequality. For a positive variable, $Y(Y>0)$, inequality is defined as the variance of $y=\log Y$. In the $\log$ scale, the variance is a scale invariant measure of inequality: multiplying the raw variable by a constant, $k Y$, adds a constant on the log scale, $k+y$, leaving the variance of $y$ unchanged. With a regression on the log scale, on $y_{i}$, the variance function coefficients are also scale invariant. Multiplying $Y$ by a constant shifts only the intercept, $\beta_{0}$, of the 
regression for the mean in the log scale. The slope coefficients for the mean and the residuals are unchanged, leaving all the variance coefficients unchanged by a change in scale. The variance of the $\log , V=V(\log Y)$, is also functionally related to several common measures of inequality including the Gini index, $G$, where

$$
G=2 \Phi\left([V / 2]^{1 / 2}\right)-1,
$$

and $\Phi(\cdot)$ is the cumulative distribution function of the standard normal distribution (Allison 1978, 874). We explore the empirical relationship between the variance of the log and the Gini index in the application below. ${ }^{2}$

We use variance function regressions to study trends in inequality by elaborating a standard variance decomposition recently applied by Lemieux (2006) to men's hourly wages. For this decomposition the data are organized in a table and each observation is assigned to a cell in the crossclassification of all covariates. With $k$ covariates, with levels $c_{1}, c_{2}, \ldots, c_{k}$, the covariates define a total of $C=c_{1} \times c_{2} \times \ldots c_{k}$ cells. For example, an earnings analysis might include covariates for education measured at three levels (say less than high school, high school, and greater than high school) and work experience (less than 5 years, 5 to 15 years, and greater than 15 years). The population could then be described by an education-byexperience table, defining $3 \times 3=9$ groups. With data configured in this way, between-group inequality describes differences across education-experience cells, and within-group inequality refers to heterogeneity within educationexperience cells.

\footnotetext{
${ }^{2}$ For log-normal data, $Y, \sqrt{V}$ is a general inequality parameter of the kind described by Jasso and Kotz (2008)
} 
More formally, for an outcome, $y_{i}=\log Y_{i}$, inequality is measured by the variance, $V$. The variance can be expressed as a weighted sum of group means and variances that yield between-group and within-group components:

$$
\begin{aligned}
V & =B+W, \\
& =\sum_{c=1}^{C} \pi_{c} r_{c}^{2}+\sum_{c=1}^{C} \pi_{c} \sigma_{c}^{2},
\end{aligned}
$$

where the $\pi_{c}$ are cell proportions, $r_{c}=\hat{y}_{c}-\bar{y}$ are deviations of the group means from the grand mean, and the $\sigma_{c}^{2}$ are the variances of $y_{i}$ for each cell.

With data at two points in time, $t=0,1$, we write the cell proportions, $\pi_{t c}$, cell residuals, $r_{t c}$, and cell variances, $\sigma_{t c}^{2}$. The change in the variance of $y$ from $t=0$ to $t=1$ can be decomposed into changes in the between-group and within-group variance. The change in the between-group variance can be written,

$$
B_{1}-B_{0}=\sum_{c=1}^{C}\left(\pi_{1 c}-\pi_{0 c}\right) r_{1 c}^{2}+\sum_{c=1}^{C}\left(r_{1 c}^{2}-r_{0 c}^{2}\right) \pi_{0 c},
$$

where the first term, $\sum_{c}\left(\pi_{1 c}-\pi_{0 c}\right) r_{1 c}^{2}$, describes a compositional effectthe change in variance due to shifts in the relative size of population subgroups, $\pi_{1 c}-\pi_{0 c}$. The second term, $\sum_{c}\left(r_{1 c}^{2}-r_{0 c}^{2}\right) \pi_{0 c}$, is the between-group effect-the change in the variance due to shifts in group means, $r_{1 c}^{2}-r_{0 c}^{2}$. The change in the within-group variance can similarly be written,

$$
W_{1}-W_{0}=\sum_{c=1}^{C}\left(\pi_{1 c}-\pi_{0 c}\right) \sigma_{1 c}^{2}+\sum_{c=1}^{C}\left(\sigma_{1 c}^{2}-\sigma_{0 c}^{2}\right) \pi_{0 c} .
$$

With these expressions, changes in the variance of $y$ can be written as the 
sum of three components:

$$
V_{1}-V_{0}=\delta_{C}+\delta_{B}+\delta_{W}
$$

where the total compositional effect reflecting shifts in the size of population subgroups is

$$
\delta_{C}=\sum_{c=1}^{C}\left(\pi_{1 c}-\pi_{0 c}\right)\left(r_{1 c}^{2}+\sigma_{1 c}^{2}\right),
$$

the between-group effect is,

$$
\delta_{B}=\sum_{c=1}^{C} \pi_{0 c}\left(r_{1 c}^{2}-r_{0 c}^{2}\right),
$$

and the within-group effect is,

$$
\delta_{W}=\sum_{c=1}^{C} \pi_{0 c}\left(\sigma_{1 c}^{2}-\sigma_{0 c}^{2}\right) .
$$

With a time series, $t=0, \ldots, T$, it is also useful to plot adjusted variances that fix at $t=0$ either the population proportions,

$$
V_{t}^{C}=\sum_{c=1}^{C} \pi_{0 c}\left(r_{t c}^{2}+\sigma_{t c}^{2}\right),
$$

the group means,

$$
V_{t}^{B}=\sum_{c=1}^{C} \pi_{t c}\left(r_{0 c}^{2}+\sigma_{t c}^{2}\right),
$$

or the group variances,

$$
V_{t}^{W}=\sum_{c=1}^{C} \pi_{t c}\left(r_{t c}^{2}+\sigma_{0 c}^{2}\right) .
$$

These adjusted variances can be interpreted as (1) the variance we would observe, $V_{t}^{C}$, if the composition of the population had remained unchanged 
from $t=0$, (2) the variance, $V_{t}^{B}$, we would observe if group means were unchanged, and (3) the variance we would observe, $V_{t}^{W}$, if within-group variances remained unchanged. In principle, neither the variance decomposition nor the adjusted variances require a regression model. As in Lemieux's (2006) decomposition, the analysis requires only cell proportions, cell means, and cell variances for all years.

Variance function regressions develop the standard decomposition in three ways. First, we are often interested in studying shifts in inequality associated with individual covariates. Indeed regression methods have often been used to decompose the change in variance in this way (e.g., Hauser and Xie 2005; Lam and Levison 1992). The extension here involves writing the residual variance as a function of covariates, allowing the researcher to isolate changes in between-group and within-group inequality associated with individual variables. Second, data may be sparse, so cells observed in some years may be unobserved in others. Regression estimates can be used to impute means and variances for empty cells, ensuring that adjusted variances are always defined. More generally, a model for cells means and variances will smooth the data, reducing the influence of outlying cells with few observations. Finally, with Bayesian posterior simulation, bounds can easily be constructed for decomposition quantities. (Posterior simulation for the usual homoscedastic regression could also be used to construct inferences for nonstandard decomposition quantities.)

The effect of predictor $x$ on changes in inequality in $y$ can be quantified with an adjusted variance that fixes a regression coefficient at its value at the baseline, $t=0$. At time $t$, we have an $n \times k$ matrix of covariates, $Z_{t}$, and a variable of interest given by the $n \times 1$ vector, $x_{t}$. With an $n \times 1$ vector 
of observations on the dependent variable, $y_{t}=\log \boldsymbol{Y}_{t}$, write a variancefunction model:

$$
\begin{aligned}
\hat{y}_{t} & =\boldsymbol{Z}_{t} \gamma_{t}+\beta_{t} \boldsymbol{x}_{t}, \text { and } \\
\log \sigma_{t}^{2} & =\boldsymbol{Z}_{t} \boldsymbol{\theta}_{t}+\lambda_{t} \boldsymbol{x}_{t} .
\end{aligned}
$$

To assess the effects of $x$ on between-group inequality, construct the adjusted variance:

$$
V_{t}^{\beta}=\sum_{c=1}^{C} \pi_{t c}\left(\tilde{r}_{t c}^{2}+\sigma_{t c}^{2}\right)
$$

With $z_{c}$ and $x_{c}$ indicating cell $c$, the adjusted between-group residual, $\tilde{r}_{t c}=$ $\tilde{y}_{t c}-\bar{y}_{t}$, is calculated from

$$
\tilde{y}_{t c}=z_{c}^{\prime} \gamma_{t}+\beta_{0} x_{c}
$$

Here, the adjusted between-group mean at time $t$ is based on all coefficients at time $t$, except for the variable of interest, $x$, where we fix the coefficient at the baseline, $t=0$. The adjusted variance, $V_{t}^{\beta}$, can be interpreted as the variance we would observe if the between-group coefficient for $x$ had remained fixed at the baseline time point, $t=0$. Similarly, an adjusted variance that describes the effect of $x$ on within-group inequality is given by,

$$
V_{t}^{\lambda}=\sum_{c=1}^{C} \pi_{t c}\left(r_{t c}^{2}+\tilde{\sigma}_{t c}^{2}\right) .
$$

where $\tilde{\sigma}_{t c}^{2}=\exp \left(z_{c}^{\prime} \theta_{t}+\lambda_{0} x_{c}\right)$. The adjusted variance, $V_{t}^{\lambda}$, can be interpreted as the variance we would observe if the effects of $x$ on within-group inequality had remained fixed at the baseline time point, $t=0$. For example, a large literature on increasing earnings inequality in the United States examines the growth in relative earnings of college graduates. Adjusted variances, $V_{t}^{\beta}$, could show the contribution of the growth in relative 
earnings of college graduates to the overall rise in inequality. Theories of labor market deinstitutionalization predict increasing earnings inequality among poorly-educated workers (e.g., McCall 2000; Sørensen 2000). Within-group inequality among low-skill workers could be studied with $V_{t}^{\lambda}$ which fixes educational difference in residual variance at the baseline time point.

The method can be generalized to study a wide range of effects. For example, interest may focus on the effects of covariates on only betweengroup or within-group inequality. In this case, just the relevant $\beta$ or $\lambda$ coefficients would be fixed at the baseline time point. Adjusted variances could also be constructed to study the effects of several covariates instead of just one.

Compositional changes can be studied by fixing the marginal distribution of individual covariates at the baseline time point. At time $t$, for each cell $c$, the covariate $x_{t}$ has marginal probability $p\left(x_{t c}\right)=p_{t c}$. For example, let $x_{t}$ be a dummy variable with a mean of .7. Then $p_{t c}=.3$ for cells in which $x_{t c}=0$, and $p_{t c}=.7$ for cells in which $x_{t c}=1$. The effects of compositional shifts in $x_{t}$ on inequality can be estimated by an adjusted set of cell proportions,

$$
\tilde{\pi}_{1 c}=\left(p_{0 c} / p_{1 c}\right) \pi_{1 c} .
$$

Again, our analysis has parallels in Lemieux's (2006) analysis of compositional effects on the residual variance of men's wages. Lemieux (2006) proposes a reweighting scheme based on the joint distribution of all covariates, not a single covariate of interest. In the current approach, adjusted cell proportions preserve the joint distribution of the population conditional on $x_{t}$, but inherit the marginal distribution of $x_{t}$ at $t=0$. The 
adjusted cell proportions are then used to form adjusted variances,

$$
V_{t}^{\pi}=\sum_{c=1}^{C} \tilde{\pi}_{t c}\left(r_{t c}^{2}+\sigma_{t c}^{2}\right) .
$$

Similar to the adjusted variances based on fixed regression coefficients, $V_{t}^{\pi}$ might be interpreted as the inequality we would observe if the marginal distribution of $x_{t}$ were unchanged from $t=0$.

\section{Application II: Decomposing Trends in Hourly Wages}

A large research literature has examined the growth in inequality in men's hourly wages (for reviews and recent contributions see Acemoglu 2002; Autor, Katz, and Kearney 2005; Lemieux 2006). In this application we study inequality in the annual wage and salary income for men aged 25 to 55 using data from the March Current Population Survey. We count only the earnings of men working full-time and year-round, and only those who report earning at least $\$ 100$ in a given year. All earnings data have been adjusted for inflation to 2001 dollars. Inequality in men's annual earnings from 1970 to 2005 is shown in Figure 1. In our variance-function analysis, inequality is measured by the variance in log annual earnings. The variance of log earnings is compared to the ratio of 90th to the 10th percentile in raw earnings. The variance and 90/10 ratios have been scaled to equal 1 in 1970. Earnings inequality increases in similar proportion with both measures. The third series in Figure 1 shows the Gini index for annual earnings. Because the Gini is a square root function of the variance of the $\log$, the variance increases more quickly than the Gini when sufficiently large. ${ }^{3}$

\footnotetext{
${ }^{3}$ Analysis of the derivative, $d G / d V$ shows that the variance increases more quickly than the Gini $(d G / d V<1)$ when $V>.075$ approximately.
} 


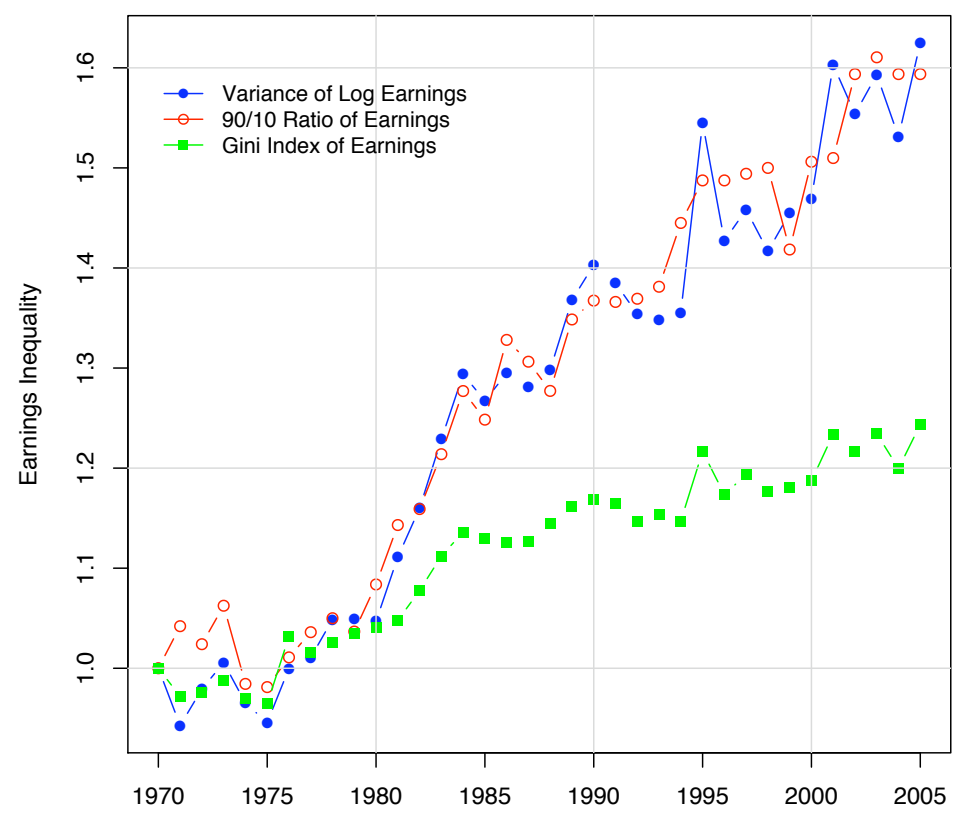

Figure 1. Trends in earnings inequality, full-time full-year men, aged 25 to 55, 1970-2005, March Current Population Survey. 
Research has focused on earnings inequality by levels of education and the growth of the residual variance in earnings. Studies of educational differences in incomes focus on the rising relative pay of college-educated workers. In 1970, college graduates earned about 35 percent more than high school graduates. By 2006, the wage advantage of college graduates had increased to 60 percent. Much of the empirical research analyzed trends in the education gradient, estimated with a regression of log earnings on years of schooling, typically controlling for experience and other covariates (Levy and Murnane 1992; Katz and Murphy 1992). The variance function analysis extends this research by calculating the contributions to overall earnings inequality of (1) between-group educational inequality in earnings, (2) within-group educational inequality in earnings, (3) the educational composition of the labor force. Groups in this analysis are defined by race, experience, and education. The analysis synthesizes the emphasis in economic research on between-group inequality by levels of education and sociological emphasis on within-group inequality among low-education workers.

With survey data on year $t(t=1970,1971, \ldots, 2005)$, a variance function regression on log earnings is written,

$$
\begin{aligned}
\hat{y}_{t i} & =\boldsymbol{x}_{t i}^{\prime} \gamma_{t}+\boldsymbol{e}_{t i}^{\prime} \boldsymbol{\beta}_{t}, \text { and } \\
\log \sigma_{t i}^{2} & =\boldsymbol{x}_{t i}^{\prime} \boldsymbol{\theta}_{t}+\boldsymbol{e}_{t i}^{\prime} \boldsymbol{\lambda}_{t}, \text { where }
\end{aligned}
$$

$\boldsymbol{x}_{t i}$ is a vector of dummy variables indicating race and ethnicity, and experience categories, and $\boldsymbol{e}_{t i}$ is a $4 \times 1$ vector of dummy variables coded for 5 educational categories: (1) less than tenth grade, (2) tenth or eleventh grade, (3) high school graduate or equivalent, (4) some college, and (5) four-year degree or more. Four adjusted variances can be constructed with 
this model to study the effects of education on the trend in earnings inequality. The first fixes between-group educational inequality in earnings at the 1970 level:

$$
V_{t}^{\beta}=\sum_{c=1}^{C} \pi_{t c}\left(\tilde{r}_{t c}^{2}+\sigma_{t c}^{2}\right)
$$

where $\tilde{r}_{t c}=\tilde{y}_{t c}-\bar{y}_{t}, \tilde{y}_{c t}=\boldsymbol{x}_{c}^{\prime} \boldsymbol{\gamma}_{t}+\boldsymbol{e}_{c}^{\prime} \beta_{1970}$, and $\boldsymbol{x}_{c}$ and $\boldsymbol{e}_{c}$ are design vectors corresponding to cell $c$ of the race by experience by education table. The second adjusted variance fixes within-group educational inequality in earnings:

$$
V_{t}^{\lambda}=\sum_{c=1}^{C} \pi_{t c}\left(r_{t c}^{2}+\tilde{\sigma}_{t c}^{2}\right)
$$

where $\log \tilde{\sigma}_{t c}^{2}=\boldsymbol{x}_{c}^{\prime} \boldsymbol{\theta}_{t}+\boldsymbol{e}_{c}^{\prime} \boldsymbol{\lambda}_{1970}$. The third adjusted variance combines the effects of educational inequalities in within-group and between-group inequalities:

$$
V_{t}^{\beta \lambda}=\sum_{c=1}^{C} \pi_{t c}\left(\tilde{r}_{t c}^{2}+\tilde{\sigma}_{t c}^{2}\right),
$$

The fourth adjusted variance fixes the marginal distribution of education at the 1970 level:

$$
V_{t}^{\pi}=\sum_{c=1}^{C} \tilde{\pi}_{t c}\left(r_{t c}^{2}+\sigma_{t c}^{2}\right),
$$

where $\tilde{\pi}_{t c}=\left(p_{1970 c} / p_{t c}\right) \pi_{t c}$ where $p_{t c}$ is the marginal probability of education in year $t$ in cell $c$.

Figure 2 shows the effects of education on the trend in US earnings inequality. The top panel compares three adjusted variances that fix education coefficients at the 1970 level. Observed inequality in earnings increases by 60 percent from 1970 to 2005, but the trend in $V_{t}^{\beta}$ indicates that inequality would have increased by only 45 percent if the educational inequality in mean earnings had remained fixed at the 1970 level. Less 
research has studied educational differences in within-group inequality (though see Juhn, Murphy, and Pierce 1993; Lemieux 2006). Trends in $V_{t}^{\lambda}$ show that differences in the within-group variance across levels of education have affected the rise in US earnings inequality in similar magnitude to the growth in between-group inequality. If the within-group and between-group effects of education are added together, trends in $V_{t}^{\beta \lambda}$ show that they explain about half the growth in US earnings inequality. Trends in $V_{t}^{\pi}$ illustrates the effect of the educational composition of the workforce (Figure $2 \mathrm{~b}$ ). The adjusted variance tracks the observed variance, indicating that the great increase in high school graduation rates and college attendance has had little net distributional effect.

Finally, Figure 3 shows the effects of trends in within-group inequality. In this case the adjusted variance is obtained by fixing all variance coefficients, $\theta$ and $\lambda$, at their 1970 level. With this adjusted variance, earnings inequality increases by just 25 percent compared to the observed increase of 60 percent. The adjusted variance indicates that 60 percent, $(60-25) / 60=.58$, of the increase in inequality in men's earnings US is associated with the growth in within-group inequality. In sum, although the effect of education on between-group inequality has been the main focus of research, the variance function analysis suggests that educational differences in within-group inequality contributes at least as much, and the overall growth in within-group explains more than half the rise inequality from 1970 to 2005.

The decomposition analysis can be taken further by reporting inferences about key quantities. Often, inferences are not provided in decomposition analyses, though sampling error is certainly present. This seems partly driven by convenience. Inferential statistics for the change 
(a) Observed and Adjusted Variances

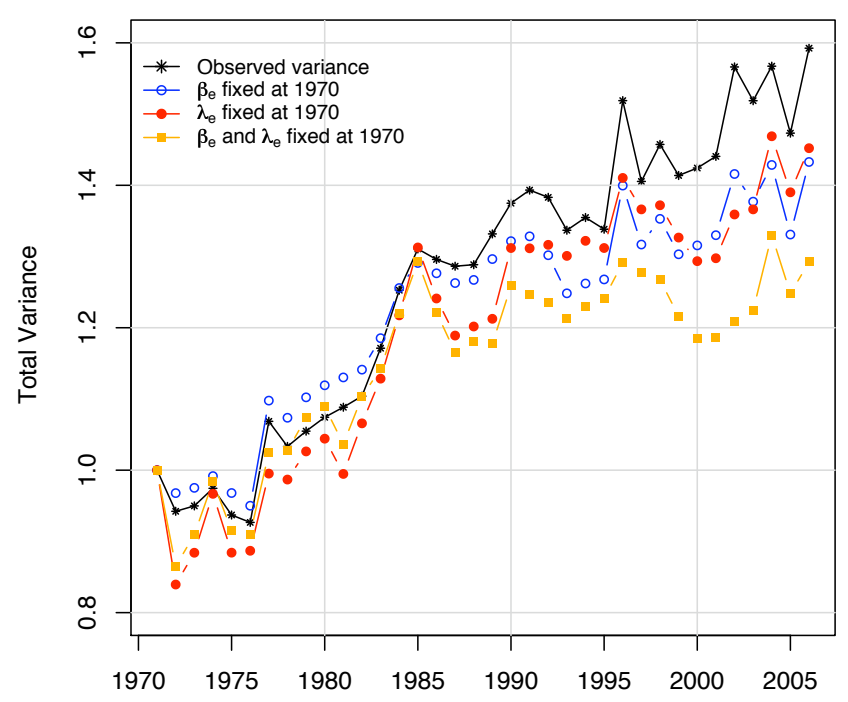

(b) Adjusted Variance with Fixed Weights

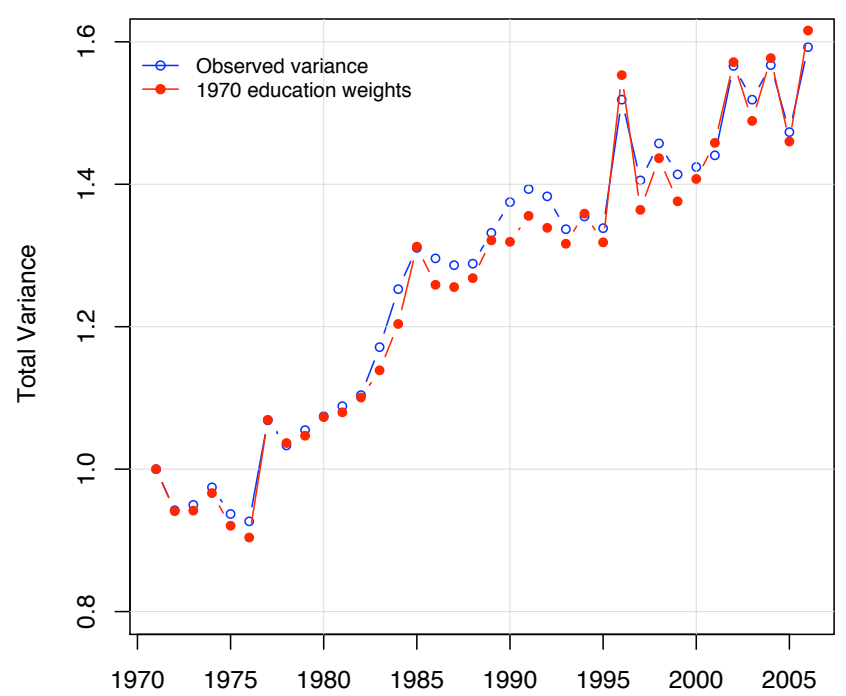

Figure 2. (a) Observed variance of log earnings, and adjusted variances fixing within-group and between-group education coefficients at 1970; (b) observed variance of log earnings and adjusted variance fixing educational attainment at the 1970, full-time full-year men, aged 25 to 55, 1970-2005. 


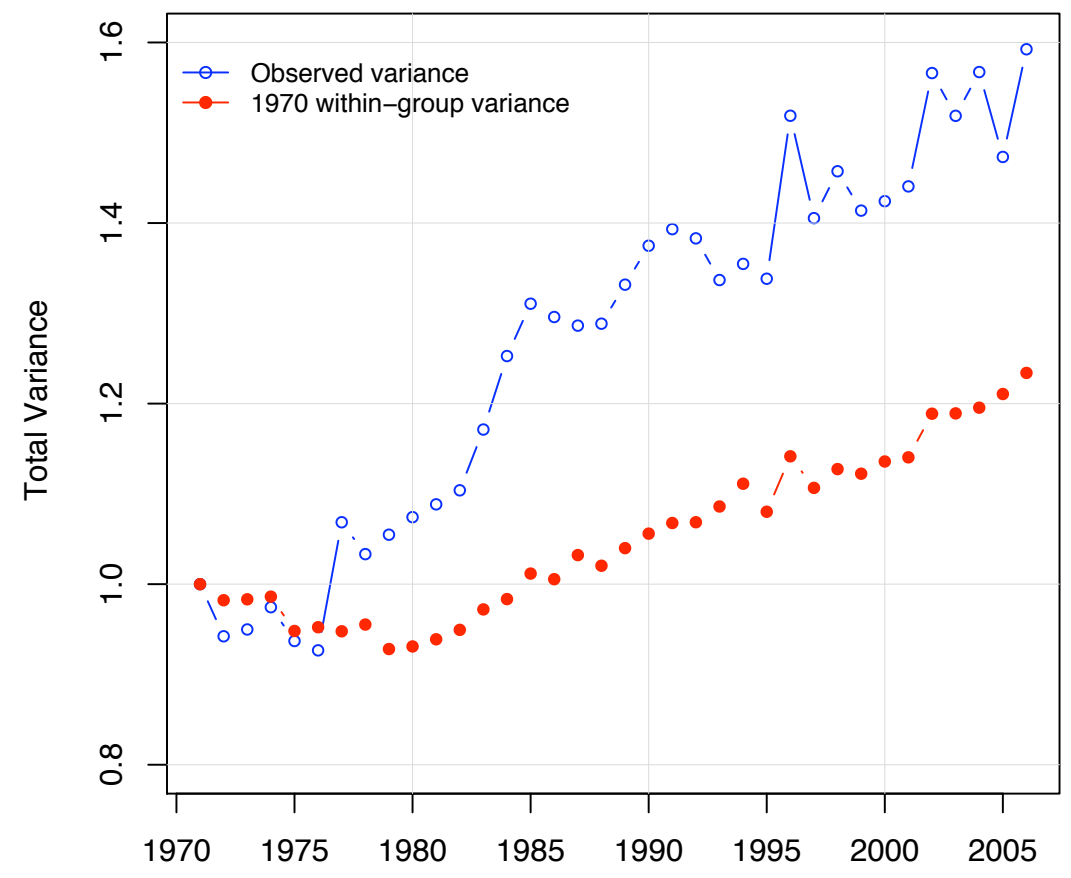

Figure 3. Observed variance in log earnings, and adjusted variance fixing variance coefficients, $\lambda$, at 1970, full-time full-year men, aged 25 to 55, 1970-2005. 
in adjusted variances are nonstandard calculations, unavailable in standard statistical packages. Still, the Bayesian analysis provides draws from the posterior distributions for all the regression coefficients. Output from posterior simulation can be used to construct standard errors and intervals for the decomposition quantities.

Draws from the posterior distribution of coefficients can be plugged into the decomposition equation to obtain standard errors and confidence intervals for the adjusted variance. In equation (1) above, we could write $V_{t}^{\beta}\left(\gamma_{t}, \beta_{1970}\right)$ indicating the dependence of the adjusted variance on 1970 education coefficients and the race and experience coefficients for year $t$. With draws from the posterior, written $\gamma_{t}^{*}$ and $\beta_{1970}^{*}$, a draw from the posterior adjusted variance is obtained with the simulated coefficients, $V_{t}^{\beta}\left(\gamma_{t}^{*}, \beta_{1970}^{*}\right)$. MCMC output consisting of $D$ draws from the posterior distributions for the mean and variance regression coefficients yields $D$ draws from the posterior adjusted variance. The standard error of the adjusted variance is estimated by the standard deviation of the $D$ draws from the posterior. Inferences for the adjusted variances, $V_{t}^{\lambda}$ and $V_{t}^{\pi}$, can be calculated in similar fashion, by plugging in the simulated values of the regression coefficients, producing posterior draws from the adjusted variance.

Table 3 reports the effects of the change in the education coefficients, $\beta$ and $\lambda$, the compositional effects of changes in educational attainment, and the effects of changes in within-group inequality on the growth in earnings inequality from 1970 to 20005. Standard errors calculated from posterior simulation are reported in parentheses. The change in variance is obtained by subtracting the observed 1970 variance from the 2005 observed and adjusted variance. To calculate inference for the change in variance, subtract 
Table 3. Observed and adjusted variances, full-time, full-year men's log annual earnings, CPS 1970-2005. Standard errors in parentheses are calculated with MCMC posterior simulation.

\begin{tabular}{lccc}
\hline \hline & & Change from & $\begin{array}{c}\text { Percent of } \\
\text { Change Explained }\end{array}$ \\
\hline Observed Variance & .481 & .179 & - \\
& $(.004)$ & $(.004)$ & \\
Adjusted variance, fixing at 1970: & & & \\
Education effects, $\beta$ & .432 & .131 & 27.2 \\
& $(.004)$ & $(.004)$ & \\
Education effects, $\beta$ and $\lambda$ & .387 & .085 & 52.5 \\
& $(.006)$ & $(.006)$ & -10.8 \\
Educational attainment & .500 & .199 & \\
& $(.006)$ & $(.004)$ & 61.4 \\
All within-group effects, $\theta$ and $\lambda$ & .371 & .069 & \\
& $(.005)$ & $(.003)$ & \\
\hline
\end{tabular}

the posterior draws from 1970 variance from the posterior draws from the 2005 variance. Results show that the standard errors are extremely small compared to the change in variance indicating that the overall growth in inequality and the growth attributable education effects and within group inequality is unlikely to be due to sampling error.

Though our analysis is based on annual earnings for full-time fullyear male workers, different data and samples may yield different results. For example, Lemieux (2006) reports large composition effects related to workforce aging in his decomposition analysis of within-group inequality in hourly wages in the Outgoing Rotation Group files of the CPS. We find little evidence of the composition effects of schooling and larger effects of schooling coefficients on between-group and within-group inequality in the March CPS annual earnings data. This divergence suggests the sensitivity of results to the range of plausible design choices. 


\section{DisCUSSION}

In this paper we proposed a variance function regression for studying the level and trend in inequality. By writing a regression model for both the mean and variance of a dependent variable, the variance function regression treats within-group, or residual, inequality as a something to be explained. In previous research on earnings, the within-group variance was interpreted to reflect the influence of returns to unobserved characteristics. Theories of inequality have also treated with-group inequality as measuring risk or insecurity. Our analysis provides a way of explaining variability in risk or insecurity in addition to the usual account of between-group inequality. We also extended the model to a variance decomposition of the change in inequality, where the variance function allows us to study the effects of covariates on both within-group and betweengroup inequality.

The model can be estimated using standard software. A two-stage estimator-consisting of a least squares fit for the mean and a gamma regression on the log squared residuals-provides accurate point estimates. Maximum likelihood estimates can be obtained by iterating between the linear regression and the gamma regression. Bayesian MCMC estimation yields draws from the full posterior distribution, producing inferences about variance decomposition.

The model was illustrated in two applications: an analysis of earnings among incarcerated respondents in the NLSY79, and an analysis of earnings inequality among US male workers from 1970 to 2005. The analysis of NLSY prisoners showed that incarceration was associated with not just reduced earnings, but also an increase in the variability of earnings. Analysis of the 35-year trend in men's earnings inequality showed that half 
of the growth in inequality is due to rising between-group and withingroup inequality by levels of education. Half of the growth in inequality is associated with the growth in within-group inequality. Changes in the educational composition of the male workforce was found to contribute very little to the growth in earnings inequality.

Variance function regressions offer a more complete model of inequality but researchers should carefully consider the model specification and measurement for this two-equation analysis. Parameterizing the mean and the variance multiplies misspecification errors. Specification errors in the model for the mean - perhaps due to omitted variables or nonlinearitiesobviously results in biased estimates of the mean coefficients. In addition, however, because the residuals are biased estimates of the true errors, coefficients for the variance will generally be biased as well, even if the variance equation is correctly specified. If the variance equation is misspecified, but the mean equation is correctly specified, the standard errors of mean regression coefficients will also be biased. However, point estimates of the mean regression coefficients will be unbiased, despite misspecification of the variance regression. ${ }^{4}$ Measurement error in the dependent variable will also affect the interpretation of the results. In particular classical measurement error will bias the intercept of the variance equation, though other coefficients will be unaffected. The variance coefficients will be biased, of course, if measurement error in the dependent variable is correlated with the independent variables. Indeed, the mean coefficients would be biased too in this situation, just as in the usual linear regression.

The current model could be extended in several ways. In the analysis

\footnotetext{
${ }^{4}$ In a correctly specified model for the mean, the errors will have zero expectation ensuring the unbiasedness of the ML estimates of $\beta$.
} 
of discrete outcomes like counts or binary variables, the mean and variance are often assumed to be functionally related. For example, a binary dependent variable, $y$, is often assumed to be Bernoulli where $E(y)=p$ and $V(y)=p(1-p)$. An overdispersion parameter is sometimes added to capture extra-Bernoulli variation, $V(y)=\phi p(1-p)$. A variance-function model with a discrete outcome might then write the overdispersion parameter, $\phi$, as a function of covariates. The model could also be extended in a Bayesian framework. The Bayesian model could be elaborated to add random components for both the mean and the variance. Where data are clustered in small areas like counties or census tracts for example, random components in the variance function would allow variability in withingroup inequality beyond that explained by the covariates. Such models could be estimated with MCMC methods for posterior simulation.

Regression analyses of inequality typically capture only differences between groups. In sociological applications, residual inequality tends to be very large in comparison to between-group inequality. The substantive significance of this large residual variance tends to be glossed either by appealing to the importance of regression coefficients or dismissing residual variance as the combined effects of measurement error and uncorrelated omitted variables. If overall inequality-the overall spread of the dependent variable-is really the main substantive interest, the variance function regression provides a useful tool, making the residual variance itself a target for analysis. 


\section{Appendix 1: Variance Function MLE's in Stata}

The following Stata code takes a dependent variable, $Y$, a local macro variable listing predictors for the mean, $\mathbf{X}$, and another listing predictors for the variance, $Z$. The code monitors the log likelihood and outputs the parameter estimates.

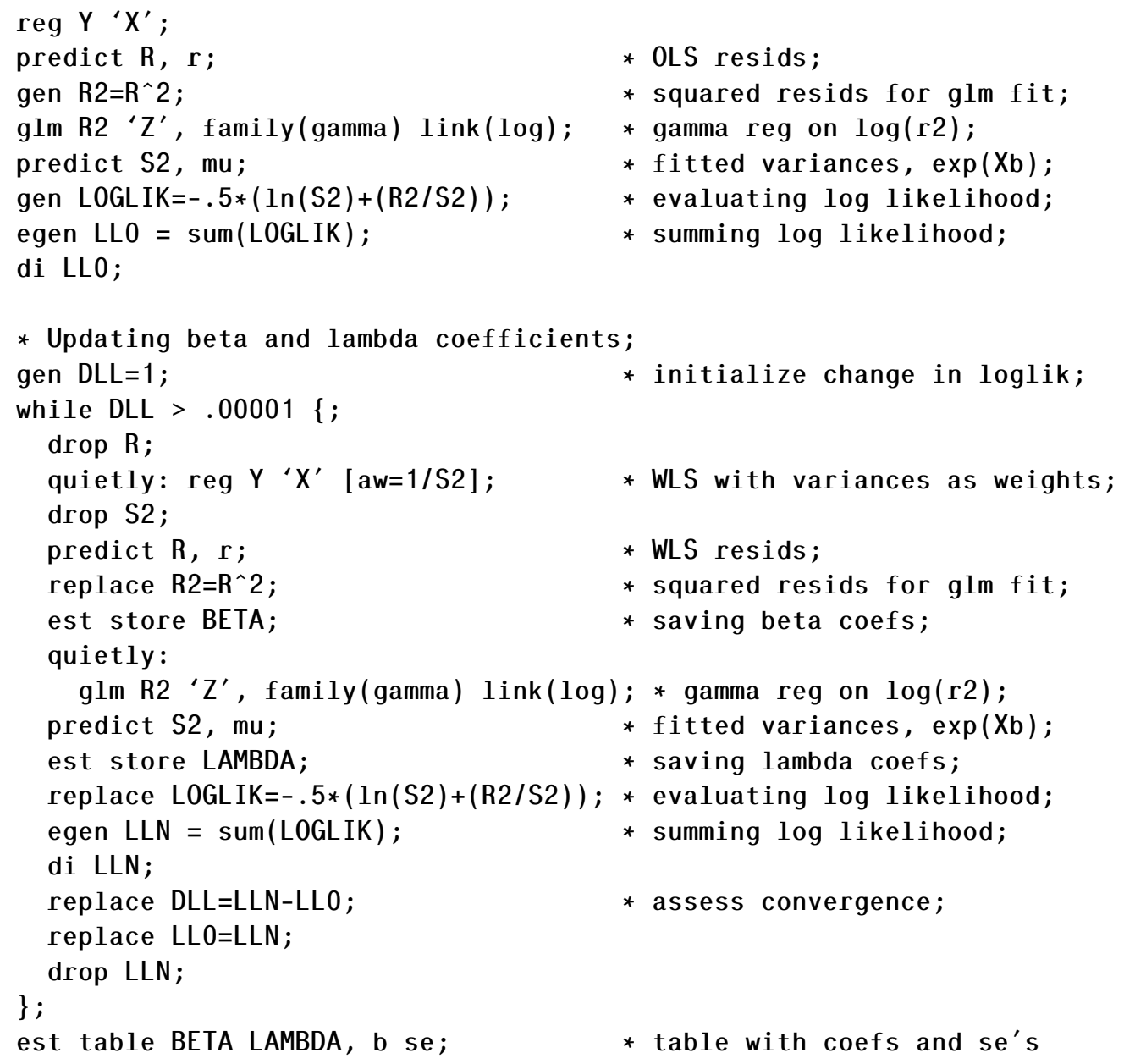




\section{Appendix 2: BUGS Code for Variance Function Regression}

The following BUGS code was used in the Monte Carlo experiment reported in Table 2. The code fits a bivariate regression with dependent variable, $y$, and a single predictor $x$, to simulate from the posterior distribution of the mean coefficients, $b 0$ and $b 1$ and the variance coefficients, lambda 0 and lambda 1.

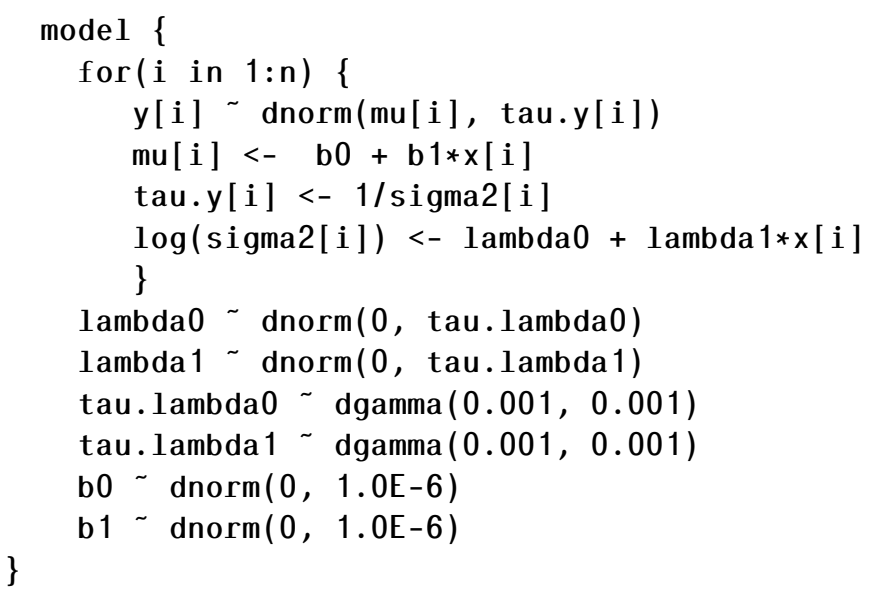




\section{REFERENCES}

Acemoglu, Daron. 2002. "Technical Change, Inequality, and the Labor Market." Journal of Economic Literature 40:70-72.

Aitkin, Murray. 1987. "Modelling Variance Heterogeneity in Normal Regression Using GLIM." Applied Statistics 36:332-339.

Allison, Paul D. 1978. "Measures of Inequality." American Sociological Review 43:865-880.

Anscombe, FJ. 1961. "Examination of Residuals." In Proceedings of the Fourth Berkeley Symposium on Mathematical Statistics and Probability, volume 1, pp. 1-36, Berkeley, CA. University of California Press.

Arden, Rosalind and Robert Plomin. 2006. "Sex Differences in Variance of Intelligence Across Childhood." Personality and Individual Differences 41:39-48.

Autor, David H., Lawrence F. Katz, and Melissa S. Kearney. 2005. "Trends in U.S. Wage Inequality: Re-Assessing the Revisionists." National Bureau of Economic Research Working Paper 11627, National Bureau of Economic Research, Cambridge, MA.

Blau, Peter M. and Otis Dudley Duncan. 1967. The American Occupational Structure. New York: Free Press.

Budig, Michelle J and Paula England. 2001. "The Wage Penalty for Motherhood." American Sociological Review 66:204- 225.

Cancio, A. Silvia, T. David Evans, and David J. Maume. 1996. "Reconsidering the Declining Significance of Race: Racial Differences in Early Career Wages.” American Sociological Review 61:541-556.

Cook, R.D. and S. Weisberg. 1983. "Diagnostics for Heteroscedasticity in Regression." Biometrika 76:1-10.

DiNardo, James, Nicole M. Fortin, and Thomas Lemieux. 1996. "Labor Market Institutions and the Distribution of Wages, 1973-1992." Econometrica 64:1001-44. 
Edwards, Jode W. and Jean-Luc Jannink. 2006. "Bayesian Modelling of Heterogeneous Error and Genotypic $\times$ Environment Interaction Variables." Crop Breeding Genetics and Cytology 46:820-833.

Harvey, Andrew C. 1976. "Estimating Regression Models with Multiplicative Heteroscedasticity." Econometrica 44:461-465.

Hauser, Seth M. and Yu Xie. 2005. "Temporal and regional variation in earnings inequality: urban China in transition between 1988 and 1995." Social Science Research 34:44-79.

Jasso, Guillermina and Samuel Kotz. 2008. "Two Types of Inequality: Inequality between Persons and Inequality Between Subgroups." Sociological Methods and Research 37:31-74.

Jencks, Christopher, Marshall Smith, Henry Acland, Mary Jo Bane, Barbara Heyns, and Stephan Michelson. 1972. Inequality: A Reassessment of the Effect of Family and Schooling in America. New York: Harper.

Juhn, Chinhui, Kevin M. Murphy, and Brooks Pierce. 1993. "Wage Inequality and the Rise in Returns to Skill." Journal of Political Economy 101:410-442.

Katz, Lawrence F. and Kevin M. Murphy. 1992. "Changes in Relative Wages, 1963-1987: Supply and Demand Factors." Quarterly Journal of Economics 107:35-78.

Kim, Chang Hwan and Arthur Sakamoto. 2008. "The Rise of IntraOccupational Wage Inequality in the United States, 1983 to 2002." American Sociological Review 73:129-157.

Kling, Jeffrey R. 2006. "Incarceration Length, Employment, and Earnings." American Economic Review 96:863-876.

Lam, David and Deborah Levison. 1992. "Age, Experience, and Schooling: Decomposing Earnings Inequality in the United States and Brazil." Sociological Inquiry 62:220-245.

Lemieux, Thomas. 2006. "Increasing Residual Wage Inequality: Composition Effects, Noisy Data, or Rising Demand for Skill?" American Economic Review 96:461-498.

Levy, Frank and Richard J. Murnane. 1992. “U.S. Earnings Levels and 
Earnings Inequality: A Review of Recent Trends and Proposed Explanations." Journal of Economic Literature 30:1333-1381.

Lewis, Matthew. 2008. "Price Dispersion and Competition with Differentialed Sellers." Journal of Industrial Economics 56:654-678.

Massey, Douglas S. 2007. Categorically Unequal: the American Stratification System. New York, NY: Russell Sage Foundation.

McCall, Leslie. 2000. "Explaining Levels of Within-Group Wage Inequality in U.S. Labor Markets." Demography 37:415-430.

Minto, Colin, Ransom A. Myers, and Wade Blanchard. 2008. "Survival Variability and Population Density." Nature 452:344-348.

Nelder, John A. and Y. Lee. 1991. "Generalized Linear Models for the Analysis of Taguchi-Type Experiments." Applied Stochastic Models and Data Analysis 7:107-120.

Pager, Devah. 2003. "The Mark of a Criminal Record." American Journal of Sociology 108:937-975.

Park, R.E. 1966. "Estimation with Heteroscedastic Error Terms." Econometrica 34:888.

Selb, Peter. 2008. "Supersized Votes: Ballot Length, Uncertainty, and Choice in Direct Legislation Elections." Public Choice 135:319-336.

Smyth, Gordon K. 2002. "An Efficient Algorithm for REML in Heteroscedastic Regression." Journal of Graphical and Compuational Statistics 11:836-847.

Smyth, Gordon K., A. Frederik Huele, and Arunas P. Verbyla. 2001. "Exact and Approximate REML for Heteroscedastic Regression." Statistical Modelling 1:161-175.

Sørensen, Aage B. 2000. "A Sounder Basis for Class Analysis." American Journal of Sociology 105:1523-1558.

Sørensen, Jesper B. and Olav Sorenson. 2007. "Corporate Demography and Income Inequality." American Sociological Review 72:766-783.

Tilly, Charles. 1998. Durable Inequality. Berkeley, CA: University of California Press.

Verbyla, A.P. 1993. "Modelling Variance Heterogeneity: Residual Maximum Likelihood and Diagnostics." Journal of the Royal Statistical Society Series B 55:493-508. 
Western, Bruce. 2002. "The Impact of Incarceration on Wage Mobility and Inequality." American Sociological Review 67:526-546.

Western, Bruce. 2006. Punishment and Inequality in America. New York: Russell Sage Foundation. 